\title{
Morphology of Planetary Nebulae-Possible Effects of Rotation on Stellar Ejecta
}

\author{
Sun Kwok \\ Department of Physics \& Astronomy, University of Calgary, Calgary, \\ Canada T2N 1 N4
}

\begin{abstract}
Planetary nebulae are formed as the result of the interaction between a slow stellar wind from the asymptotic giant branch progenitor and a later-developed fast outflow from the central star. Many of the morphological and kinematic properties of planetary nebulae have been successfully explained by this interacting stellar winds model. The observed diverse morphologies of planetary nebulae can also be understood if the slow wind is not spherically symmetric. However, new observational features such as collimated outflows and multi-polar lobes suggest that the fast wind may be non-isotropic and time variable. The possible roles of magnetic fields and rotation may play in the formation of these features are discussed.
\end{abstract}

\section{Introduction}

Although the existence of planetary nebulae (PN) has been known for over 200 years and the PN phenomenon has been identified as a phase in the late stages of stellar evolution since 1956 (Shklovsky 1956), the formation mechanism of PN has remained a mystery until the late 1970s. Most of the ejection theories proposed in the 1960's and 70's relied on various forms of instabibilities (dynamical, pulsational, and thermal), but none of these theories were able to eject the right amount of mass over a right time scale. At the same time, advances in infrared and millimeter-wave astronomy have revealed that the progenitors of PN, asymptotic giant branch (AGB) stars, are losing mass at a high rate (cf. Kwok 1987), and it became apparent that the extensive circumstellar envelopes (CSE) built up by such mass loss must have an effect on the formation of PN.

PN have definite morphologies, and have higher densities and expansion velocites than the CSE of AGB stars. In order to compress, accelerate, and shape the CSE into a PN, a separate mechanism is necessary. The solution was found in the interacting stellar winds (ISW) model (Kwok, Purton, \& FitzGerald 1978, Kwok 1982). The ISW model suggests that a PN is formed by a snow-plow process in which the remnant AGB CSE is compressed into a shell by a later-developed fast wind. In addition to the well-observed shell, the ISW model predicts the following components: a fast wind from the central star, a low-density halo representing the unshocked AGB wind, and a high-temperature bubble representing the shocked fast wind. With the launch of the IUE satellite in 1978, P Cygni profiles implying wind velocities of several thousands $\mathrm{km}$ 
$\mathrm{s}^{-1}$ were found in many central stars of PN (Heap et al. 1978), confirming that fast winds are indeed common in PN. Evidence for the existence of haloes outside the PN shells were found by CCD imaging, and by the detections of dust and molecular envelopes by infrared (e.g. IRAS) and millimeter-wave observations. Extended diffuse X-ray emission from the hot bubble was first detected by ROSAT and by CHANDRA (Kastner et al. 2000, Chu et al. 2001). A review of our modern understanding of the PN phenomenon can be found in Kwok (2000).

\section{The Dynamics of Interacting Winds}

The ISW model suggests that a PN is not only radiatively interacting with the nebula, but dynamically interacting as well. The appearance and the structure of PN therefore reflect the coupled dynamical and ionizational evolution of the nebula. Spherical-symmetric dynamical models incorporating the evolution of the central star reveal changing density, velocity, and temperature structures as the nebula evolves, and are able to explain the complex multi-shell (rims, shells, crowns and haloes) structures observed in PN (Schönberner \& Steffen 2003).

However, many PN are not spherically symmetric. Of particular interest is the class of bipolar nebulae that show a pair of lobes emerging from a dust torus (e.g. NGC 2346, NGC 6302). Deep CCD imaging has revealed the some elliptical nebulae are in fact bipolar (e.g. NGC 650-1, Sh 1-89, and SaWe 3). Projection effects also could be the cause of bipolar nebulae being seen to have ring-like shapes (e.g. NGC 3132, Monteiro et al. 2000).

The possibility that asymmetric PN can be produced by the ISW process if the AGB wind has an asymmetric density profile was discussed by Balick (1987). Two-dimensional numerical models have shown that a variety of PN morphologies can be produced by the ISW model (e.g. Frank et al. 1993, Frank \& Mellema 1994, Mellema \& Frank 1995).

\section{Unsolved Problems in PN Morphology}

Recent observations, in particular high-resolution images obtained with the $H S T$, have discovered new microstructures which were not predicted by the ISW model. These features include:

- FLIERS and jets: Fast Low-Ionization Emission Regions are pairs of small, bright knots of low excitation gas found along the major axes of PN (e.g. NGC 6826). Linear structures (jets) can be seen in two of the corners of the [NII] image of NGC 6543. The existence of these features suggests that the fast outflow could be collimated rather than spherical.

- Rings and arcs: concentric circular arcs have been observed in both PN (e.g. Hb 5, NGC 6543, NGC 7027) and proto-planetary nebulae (PPN, e.g. AFGL 2688, IRAS 17150-3224). These arcs are of almost perfectly circular in shape, and have relatively uniform separations of $\sim 10^{2} \mathrm{yr}$. Similar arcs have been detected in the carbon star IRC+10216, suggesting that these features originate in the AGB phase. The coexistence of these 

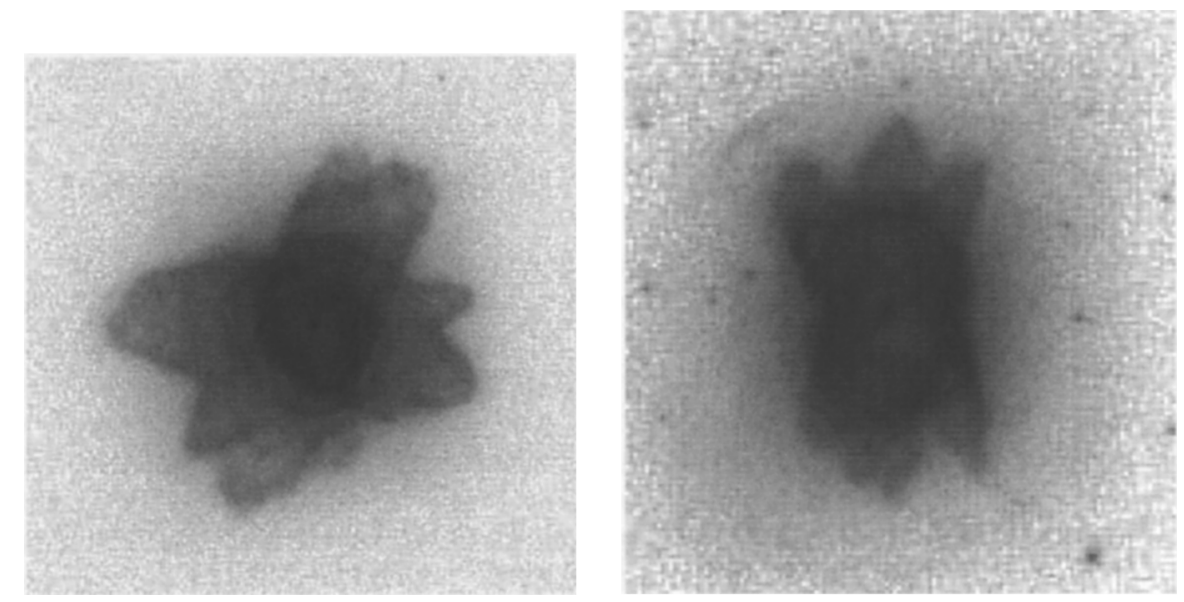

Figure 1. HST $\mathrm{H} \alpha$ images of He2-47 (left) and M1-37 (right) showing a multi-polar structure.

perfectly circular features with bipolar lobes suggests that the arcs are projections of undisturbed spherical shells on the sky (Kwok et al. 2001).

Two-dimensional rings perpendicular to the bipolar axis have been found in several PN, including MyCn18 (Sahai et al. 1999a) and NGC 6881 (Kwok, Su, \& Sahai 2003). The origin of these rings is not understood.

- Point-symmetric structures: point-symmetric pairs of knots in an S-shape structure, or sometimes referred to as bipolar, rotating, episodic jets (BRET), have been seen in a number of PN (e.g. K3-35, Aaquist \& Kwok 1989; Fleming 1, López et al. 1993; KjPn8, López et al. 1995; NGC 6884, Miranda et al. 1999). Some PN have been found to have more than one polar axis or have a point-symmetric structure (Figures 1 and 2). These morphologies suggest that the outflow direction has changed with time (e.g. NGC 2440, López et al. 1998; M1-37 and He2-47, Sahai 2000).

- Collimated outflows: some PN and PPN (e.g. He 3-401, Sahai et al. 1999b, Fig. 3a) have extreme bipolar (cylindrical) shapes, suggesting that their morphology is shaped by a collimated outflow. The direct imaging of bipolar lobes emerging from a circumstellar disk in the PPN IRAS 17106-3046 (Kwok et al. 2000, Fig. 3b) suggests that disks could play a role in the collimation of the bipolar flows.

Further examples of $\mathrm{PNe}$ with exotic morphologies can be found in Kwok (2001, 2002).

\section{The Effects of Rotation on Collimated Outflows and Point-Sym- metric Structures}

While an asymmetric AGB wind can lead to bipolar structures through ISW shaping, the existence of highly collimated outflows suggests that the fast wind 

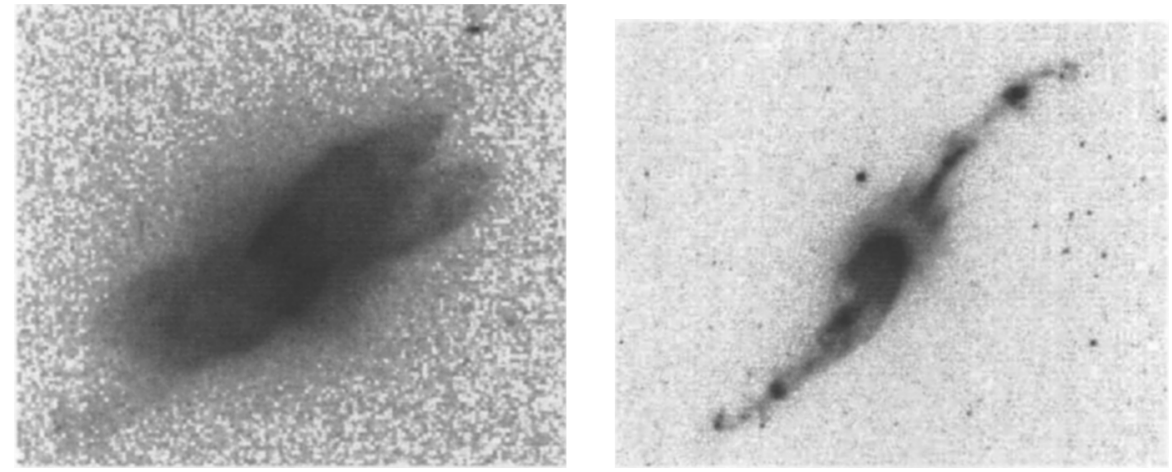

Figure 2. HST [NII] images of He2-447 (left) and He3-1475 (right). He2-447 has a multi-polar structure and He3-1475 shows a S-shape structure.
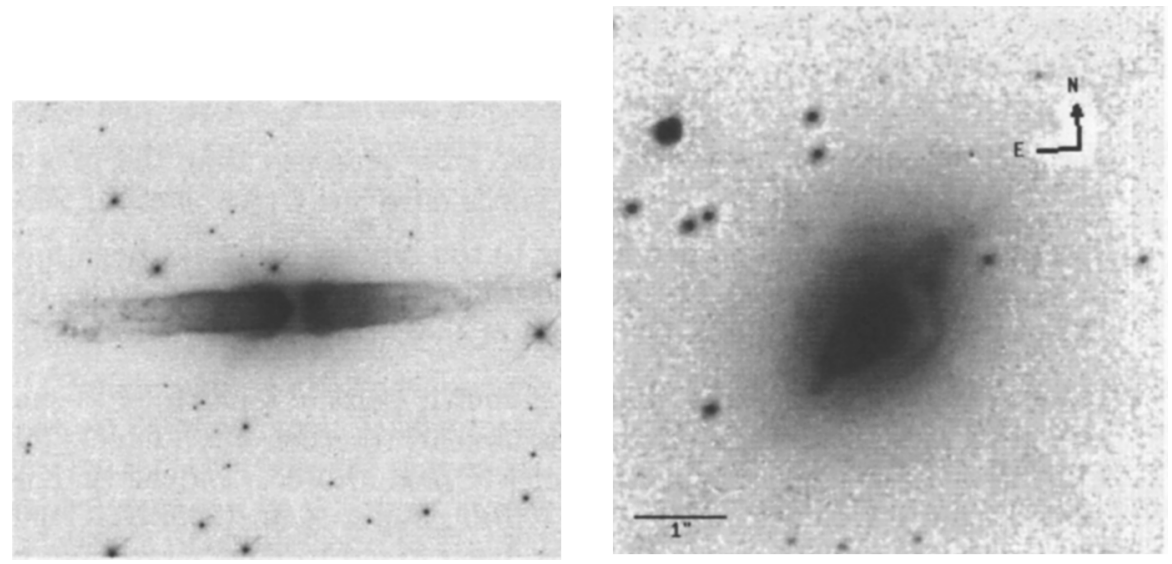

Figure 3. HST V-band images of He3-401 (left) and IRAS 17106-3046 (right). Hen 3-401 shows a highly-collimated outflow and a collimating disk can be seen in IRAS 17106-4046.

can also be non- isotropic. The BRET phenomenon points to a fast wind whose mass loss rate and velocity are time variable in magnitude and in direction. Possible origins include warped accretion disks (Livio \& Pringle 1996), precession of wide binaries (García-Segura 1997), or rotating central stars with magnetic fields (Chevalier \& Luo 1994, García-Segura et al. 1999).

The effects of rotation on mass loss through equatorial density enhancements have been studied by various authors (Bjorkman \& Cassinelli 1993, Ignace et al. 1996), and hydrodynamical simulations of such processes have been made by Owocki et al. (1996). In the context of young stars, the combined effects of magnetic field and rotation have been extensively studied (Shu 1994). In a rapidly rotating AGB star, a dipolar magnetic field can take on a torodial shape. When a radiatively driven fast wind interacts with the AGB wind, the toroidal magnetic field can focus the material into its axis and produce a collimated outflow (Blackman et al. 2001). If this wind axis is tilted w.r.t the axis of 
the equatorial torus (created by the AGB wind), then a point symmetric nebula can be the result (García-Segura \& López 2000).

\section{Rotation and Concentric Arcs}

The detection of multiple concentric arcs in PN and PPN is one of the most unexpected discovery of $H S T$ observations of PN. The uniform spacings between the arcs correspond to time intervals of several hundred years, which are too short for thermal pulse and too long for pulsation. Theories proposed to explain the arcs include dynamical instability in the wind (Deguchi 1997) and mass loss modulated by the binary orbital period (Mastrodemos \& Morris 1999). Soker $(2000,2002)$ suggested that these arcs are the result of periodic dust formation due to the magnetic cycle. If this is the case, the observed time interval between arcs implies a magnetic cycle of 200-1000 yr. Assuming rotation periods of 10$100 \mathrm{yr}$ for AGB stars, this suggests a magnetic to rotation cycle ratio of $\sim 10$. An alternate proposal suggests that the arcs are formed by pressure oscillations as the result of cyclic polarity reversal of the magnetic field (García-Segura et al. 2001). In this case, the observed arc intervals would correspond to half of the magnetic cycle.

\section{Rotation on the AGB}

The above scenarios require that an AGB to rotate rapidly. For example, the existence of a magnetic field would require dynamo generation from a rapidly rotating core. Is it possible that the rotational rate of stars increases as the evolve up the AGB? When the Sun condensed from the solar nebula, most of the angular momentum was distributed to the planets. When the Sun enters the AGB, it will expand and engulf the inner planets, absorbing their angular momentum in the process (Soker 2001). The Earth, e.g. will release angular momentum of $3 \times 10^{47} \mathrm{~g} \mathrm{~cm}^{2} \mathrm{sec}^{-1}$ and can significantly spin up the AGB Sun.

\section{Summary}

Recent HST observations show that the fast wind in PN can be highly collimated, and rotation and magnetic fields may play a role in the collimation of such winds. The observations of collimated winds and concentric arcs in PN open the possibility of determining the rotation rates in the cores and envelopes of AGB stars.

Acknowledgments. This work is supported by the Natural Sciences and Engineering Research Council of Canada and the award of a Killam Fellowship by the Canada Council for the Arts.

\section{References}

Aaquist, O.B., \& Kwok, S. 1989, A\&A 222, 227

Balick, B. 1987, AJ94, 671 
Bjorkman, J.E., \& Cassinelli, J.P. 1993, ApJ 409, 429

Blackman, E.G., Frank, A., \& Welch, C. 2001, ApJ 546, 288

Chevalier, R.A., \& Luo, D. 1994, ApJ 421, 225

Chu, Y.-H., Guerrero, M.A., Gruendl, R.A., Williams, R.M., \& Kaler, J.B. 2001, ApJ, $553, \mathrm{~L} 69$

Deguchi, S. 1997, in IAU Symp. 180: Planetary Nebulae, H.J. Habing H.J.G.L.M. Lamers (eds.), Kluwers, p. 151

Frank, A., \& Mellema, G. 1994, A\&A 289, 937

Frank, A., Balick, B., Icke, V., \& Mellema, G. 1993, ApJ 404, L25

García-Segura, G. 1997, ApJ 489, L189

García-Segura, G., \& López, J.A. 2000, ApJ 544, 336

García-Segura, G., López, J.A., \& Franco, J. 2001, ApJ 560, 928

García-Segura, G., Langer, N., Rózyczka, M., \& Franco, J. 1999, ApJ 517, 767

Heap, S. R. et al., 1978, Nature 275, 385

Ignace, R., Cassinelli, J.P., \& Bjorkman, J.E. 1996, ApJ 459, 671

Kastner, J.H., Vrtilek, S.D., \& Soker, N. 2001, ApJ 550, L189

Kwok, S. 1982, ApJ 258, 280

Kwok, S. 1987, Phys. Reports, 156, 111

Kwok, S. 2000, The Origin and Evolution of Planetary Nebulae, Cambridge University Press

Kwok, S. 2001, Cosmic Butterflies, Cambridge University Press

Kwok, S. 2002, Mercury, 31, 24

Kwok, S., Purton, C.R., \& FitzGerald, M. P. 1978, ApJ 219, L125

Kwok, S., Su, K. Y. L., \& Hrivnak, B.J. 1998, ApJ 501, L117

Kwok, S., Hrivnak, B.J., \& Su, K.Y.L. 2000, ApJ 544, L149

Kwok, S., Su, K.Y.L., \& Stoesz, J. 2001, in Post-AGB Objects as a Phase of Stellar Evolution, eds. R. Szczerba and S.K. Górny, Kluwer, p. 115

Kwok, S., Su, K.Y.L., \& Sahai, R. 2003, in IAU Symp. 209, in press

Livio, M., \& Pringle, J.E. 1996, ApJ 465, L55

López, J.A., Meaburn, J., \& Palmer, J.W. 1993, ApJ 415, L135

López, J.A., Vázquez, \& Rodríguez, L.F. ApJ 455, L63

López, J. A., Meaburn, J., Bryce, M., \& Holloway, A. J. 1998, ApJ 493, 803

Mastrodemos, N., \& Morris, M. 1999, ApJ 523, 357

Mellema, G., \& Frank, A. 1995, MNRAS 273, 401

Miranda et al. 1999, ApJ 117, 1421

Monteiro, H., Morisset, C., Gruenwald, R., \& Viegas, S.M. 2000, ApJ 537, 853

Owocki, S.P., Cranmer, S.R., \& Gayley, K.G. 1996, ApJ 472, L115

Sahai, R. 2000, ApJ 537, L43

Sahai, R. et al. 1999a, AJ 118, 468

Sahai, R., Bujarrabal, V., \& Zijlstra, A. 1999b, ApJ 518, L115

Schönberner, D., \& Steffen, M. 2003, IAU Symp. 209, in press

Shklovsky, I. 1956, Astr. Zh., 33, 315

Shu, F., Najita, J., Ostriker, E., Wilkin, F. Ruden, S. \& Lizano, S. 1994, ApJ 429, 781

Soker, N. 2000, ApJ 540, 436

Soker, N. 2001, MNRAS 324, 699

Soker, N. 2002, ApJ 570, 369 